\title{
Does charge matter in high-energy collisions of black holes?
}

\author{
Gabriele Bozzola* \\ Department of Astronomy, University of Arizona, Tucson, AZ, 85721, USA
}

(Dated: February 14, 2022)

\begin{abstract}
We perform numerical-relativity simulations of high-energy head-on collisions of charged black holes with the same charge-to-mass ratio $\lambda$. We find that electromagnetic interactions have subdominant effects already at low Lorentz factors $\gamma$, supporting the conjecture that the details of the properties of black holes (e.g., their spin or charge) play a secondary role in these phenomena. Using this result and conservation of energy, we argue these events cannot violate cosmic censorship.
\end{abstract}

Introduction High-energy collisions of black holes are excellent laboratories to probe general relativity and to study the theory under extreme conditions (for reviews, see relevant sections in [1,2]). Due to its highly dynamical nature, the problem is best approached with numerical calculations, as the ones that opened this line of research in 2008 [3, 4]. Since then, studies have explored most of the possible variables (mass, impact parameter, spin [3-13]), with the noticeable exclusion of charge (electric, or associated to a generic U(1) field). In this Letter, we tackle this long overdue problem and present general-relativistic simulations of head-on collisions of black holes with the same charge.

One of our main objectives is to test whether "matter matters" in the ultra-relativistic regime [14-16]. According to this idea, the details of the properties of bodies (e.g., their spin, or composition) are irrelevant in collisions with high center-of-mass energy. The conjecture originates from considering that ultra-relativistic mergers are dominated by the kinetic energy, so the details of the interaction are unimportant. This has been verified numerically for spinning $[8,17]$ and non-spinning black holes $[3,4,7]$, as well as for boson fields [18], perfect fluids [19, 20], and plane waves [21]. The problem has also been studied in higher dimensions [11-13, 22, 23], where, in case of $\mathrm{AdS}_{5}$, it is relevant for gauge-gravity dualities. This conjecture is also at the basis of Monte Carlo event generators [24-26] for microscopic black holes in particle accelerator. Here, we test this hypothesis for black holes with charge, parameter that has not been considered so far.

Our second goal is to check if it is possible to form naked singularities with ultra-relativistic collisions, verifying whether the cosmic censorship conjecture holds. Testing this has been a recurring theme in this line of research (e.g. [3, 5, 13, 23]), but no violation has been found so far in four-dimensional spacetimes. High-energy collisions of charged black holes are a particularly interesting setting to investigate this idea because charge is another way, together with spin, to reach black-hole extremality. Kerr-Newman spacetimes with too much charge and/or spin compared to their mass do not have horizons [27], so overcharging or overspinning a black hole would be a way to form a naked singularity. Because of the emission of energy, ultra-relativistic collisions might lead to conditions in which the remnant would be "over-extremal", and create a naked singularity. In the case of spinning black holes, this is avoided by radiating away the excess angular momentum. However, charge is conserved and cannot be radiated away, constituting a significant difference compared to spin. Moreover, if charge does not matter, the colliding black holes will always merge and will not repel due to electrostatic interaction. So, if the formation of naked singularities is avoided, it is interesting to understand how this is achieved.

This Letter focuses on testing whether charge is important in the context of high-energy collisions and whether naked singularities can form in this environment. Our goal is not to perform a high-precision study, which would require extreme numerical resolution and sophisticated initial data (see, e.g. [6, 28]), but we aim to describe the general features of the phenomenon. Our main conclusion is that we find evidence that, even at low value of the boost factor $\gamma$, important gauge-independent quantities do not depend on the charge, supporting the idea that charge does not matter in ultra-relativistic collisions. Having found no evidence that all the kinetic energy in the system can be radiated away, we argue that ultrarelativistic collisions of black holes with the same charge do not form naked singularities. These conclusions are robust despite the overall accuracy of our simulations of order $10 \%$. In general, our full general relativistic calculations show that the problem can be well-understood with simple semi-classical arguments, which we present below.

The Letter is structured as follows. First, we describe our theoretical and numerical setup. Then, we report the results and our interpretation, and finally, we give some concluding remarks. We use Gaussian units with $G=c=4 \pi \epsilon_{0}=1$, and we report results in terms of $M=M_{1}+M_{2}$, where $M_{1}$ and $M_{2}$ are the individual Christodoulou masses [29, 30].

Setup We solve the Einstein-Maxwell equations in the $3+1$ decomposition of the spacetime [31, 32] (see also [33-35]) for head-on collision of equal-mass, equal-charge black holes with charge-to-mass-ratio $\lambda \in$ $\{0,0.2,0.4,0.6,0.8\}$ and initial linear momentum $P / M \in$ $\{0.2,0.4,0.6\}$. We use the Einstein Toolkit $[36,37]$ for 
the numerical integration and kuibit [38] for the analysis. We adopt the same setup as in [39], where we provide a more in-depth discussion. Note that, with the exception of kuibit and TwoChargedPunctures (see below), we use the same computational tools that have been extensively employed in this line of research (e.g. [3, 5$8,10,11,17,40,41])$.

We generate constraint-satisfying initial data with TwoChargedPunctures [30] for two black holes with masses [29, 30] $M_{1}=M_{2}=0.5 M$ and charge-to-mass ratio $\lambda_{1}=\lambda_{2}=\lambda$. The two punctures are aligned along the $z$ axis with an initial separation of $150 \mathrm{M}$. In the limit of infinite separation of in the case of isolated black holes, TwoChargedPunctures [30] reduced to Reissner-Nordström in isotropic coordinates. The boost factor is controlled by the Bowen-York momentum $P$, an input parameter in TwoChargedPunctures, which is equal to the Arnowitt-Deser-Misner (ADM) [31, 32] linear momentum for a case of a single black hole [30] and corresponds to Lorentz factor of $\gamma=\sqrt{1+4 P^{2} / M^{2}}$. TwoChargedPunctures employs the conformal-tracelesstraverse approach [30, 42-44], extending what is done by the well-known TwoPunctures [45] pseudo-spectral solver for the uncharged case. In particular, the code assumes conformal flatness and Reissner-Nordström electromagnetic fields. This leads to "junk" radiation, especially in the electromagnetic sector, that can be up to a few percent of the total energy. The initial separation is large enough that we can isolate the real signal from the spurious one (see also Supplemental Material).

We evolve the spacetime and electromagnetic fields with the Lean and ProcaEvolve codes [46-49]. Lean implements the Baumgarte-Shapiro-Shibata-Nakamura (BSSN) formulation of Einstein's equation [50, 51] and the moving puncture approach, while ProcaEvolve evolves the electromagnetic vector potential to maintain the magnetic field divergenceless and has a constraintdamping scheme for the Gauss constraint. We use the Lorenz gauge for the electromagnetic potential, the $1+\log$ and $\Gamma$-freezing slicing conditions for the lapse function and shift vector [52-54].

The simulations are on Carpet [55] Cartesian grids with octant symmetry, with two centers of refinement (one tracking the puncture, and the other fixed in the center) and 13 levels. The outer boundary is placed at least at $600 \mathrm{M}$, where it is not in causal contact with the inner part of the grid throughout the duration of the simulations. We use the continuous Kreiss-Oliger dissipation introduced in [39].

Since the size of the horizons depends on the chargeto-mass ratio $\lambda$, we change the resolution to ensure that the black holes are always resolved with at least 80 points. We estimate the initial horizon radius as if it was a Reissner-Nordström black hole in isotropic coordinates [30] and set the finest grid spacing to $\Delta x_{\text {finest }}=$ $\sqrt{1-\lambda^{2}} / 320 M .^{1}$ Depending on the charge, this can lead to resolutions up to $M / 550$.

We find that our simple prescription for the grid resolution is effective in properly resolving the black holes. We locate apparent horizons with AHFinderDirect [56], and compute their properties with QuasiLocalMeasuresEM [30], an extension of QuasiLocalMeasures [57] for full Einstein-Maxwell theory [58-60]. At the level of the initial data, we find that the horizons are coordinate ellipsoids covered by at least 40 points along the semi-minor axis. Then, the horizons expand and for most of the simulation our grid resolves the semi-minor axis with at least 120 points. The merger remnant is resolved even better. As a result, the quasilocal properties are well-behaved in all our simulations (e.g., charge is conserved at better than $0.6 \%$ ).

We extract radiation with the Newman-Penrose formalism $[22,49,61]$ at finite extraction radii ranging from $80 M$ to $200 M$. We note that, while the properties of the horizons are remarkably stable, interpolation across several refinement boundaries and the truncation error in the wave zone lead to noisy electromagnetic waves (see Supplemental Material).

Results The main conclusion from our simulations of high-energy head-on collisions of black holes is that charge does not matter for a number of gaugeindependent quantities. Before we present our results in detail, we define quantitatively what we mean by "charge does not matter". In Newtonian physics, the problem of two charged point masses is mathematically equivalent to the purely gravitational one upon rescaling of $G$ by a factor $\left(1-\lambda^{2}\right)$. This simple scaling is surprisingly effective in predicting results of fully general-relativistic calculations [39-41, 62]. Therefore, if charge mattered, we would expect most results (e.g., amplitude of $\Psi_{4}$ ) to vary with factors $\left(1-\lambda^{2}\right)$ for varying $\lambda$ and fixed $P$. Conversely, if charge did not matter, all the results should become approximately the same within our error (see, Supplemental Material).

We demonstrate that charge has negligible influence in the dynamics of high-speed mergers by discussing some key properties of the gravitational waves and of the horizons. In Fig. 1, we present the real part of the dominant mode of the Newman-Penrose scalar $\Psi_{4}(l=2$, $m=0$ ) for simulations with fixed Bowen-York momentum $P=0.4 M$ and varying charge-to-mass ratios $\lambda$. We do not apply any time-shift or any other transformation to align the signals. The good alignment indicates that charge does not have a strong impact in the event (compare this with Fig. 5 in [40], where signals had to be

\footnotetext{
${ }^{1}$ In isotropic coordinates, the horizon radius for a ReissnerNordström black hole with mass $M_{1}=0.5 M$ and charge $Q_{1}=$ $\lambda M_{1}$ is $\sqrt{1-\lambda^{2}} / 4 M$.
} 
scaled by the factor $1-\lambda^{2}$; see also Supplemental Material for a more detail comparison). We find the same properties as in the uncharged case [3]: there is a precursor signal, a main burst after the formation of the apparent horizon, and the ringdown. The time of formation of the common apparent horizon (vertical dashed line) is nearly independent of the charge and the peak of the signal occurs always approximately $15 \mathrm{M}$ after this time. The total energy lost by gravitational and elec-

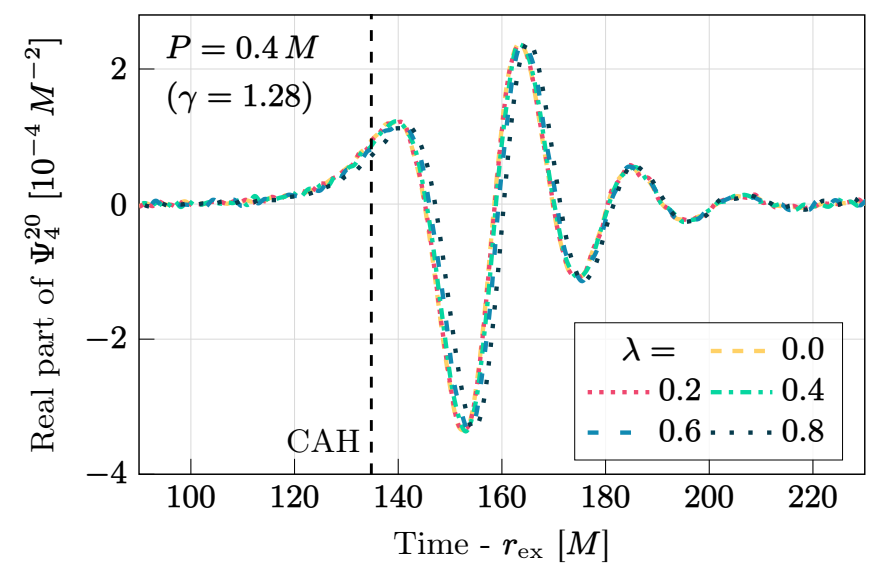

FIG. 1. Real part of the dominant multipolar component of the Newman-Penrose scalar $\Psi_{4}(l=2, m=0)$ for simulations with different charge-to-mass ratio $\lambda$ as extracted at radius $r_{\mathrm{ex}}=131.430 \mathrm{M}$. Note that no time-shift was applied to the signals: the almost identical alignment indicates that charge has a negligible influence in these collisions. The time of formation of the horizon is also nearly insensitive to the value of charge.

tromagnetic waves is reported in Fig. 2. Collisions from zero initial momenta were studied in [40], where it was found that there is a significant contribution from electromagnetic fields to the energy radiated (up to $25 \%$ ). Instead, we never find large amounts of electromagnetic waves in our simulations, and almost all the energy is lost through gravitational waves. Fig. 2 shows how all our simulations at fixed $P$ radiate the same amount of energy irrespective of $\lambda$ (within our error, see Supplemental Material). In Fig. 2, we also plot the estimate of the energy lost in ultra-relativistic collisions obtained in the Zero-Frequency-Limit (ZFL) [63], which has been shown to be a good approximation to the fractional energy lost $E_{\mathrm{GW}} / M_{\mathrm{ADM}}$ for collisions in absence of charge $[3,6]$. According to this formalism, $E_{\mathrm{GW}} / M_{\mathrm{ADM}}$ scales as:

$\frac{E_{\mathrm{GW}}}{M_{\mathrm{ADM}}}=E_{\infty}\left(\frac{1+2 \gamma^{2}}{2 \gamma^{2}}+\frac{\left(1-4 \gamma^{2}\right) \ln \left(\gamma+\sqrt{\gamma^{2}-1}\right)}{2 \gamma^{3} \sqrt{\gamma^{2}-1}}\right)$,

where $E_{\infty}$ is the energy lost for infinitely boosted black holes, which has numerically been calibrated to be approximately 0.13 . Our simulations also find a good level of agreement with the ZFL estimate.

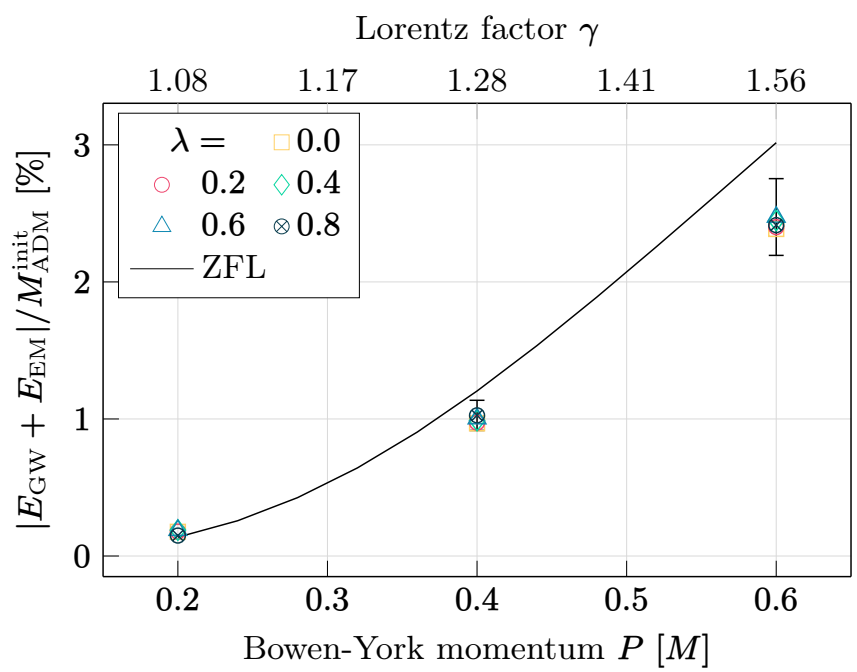

FIG. 2. Total energy lost by gravitational and electromagnetic waves normalized to the initial ADM mass. At any given Bowen-York momentum $P$, the energy lost for different values of $\lambda$ is the same (within our error, see Supplemental Material). The black line is the Zero-Frequency-Limit (ZFL) prediction [63] (Eq. (1) with $E_{\infty}=0.13$ ), which has been shown to be accurate for uncharged collisions $[3,6]$.

Finally, we consider the remnant properties. We find that the fractional difference of the quasi-local mass of the final black hole between the charged and uncharged cases is always below $1 \%$. This implies that the mass of the remnant does not depend on $\lambda$ at the level of our accuracy (see Supplemental Material). Note, however, that apparent horizons are not completely gauge invariant as they depend on the spacetime slicing.

Our simulations demonstrate that even with small boosts $(\gamma \approx 1.1)$ charge does not matter in the dynamics of the event and in a number of gauge-independent quantities, or if it did, it would do so only at the percent level (contrarily to what happens for $\gamma=1[40]$ ). We can build intuition on why this happens with the following qualitative semi-classical argument. Consider a head-on collision of two black holes with mass $\mathcal{M}$, charge $\mathcal{Q}=\lambda \mathcal{M}$, Lorentz factor $\gamma$, and infinite initial distance. Initially, the interaction is negligible and the motion is completely determined by the initial velocity. The separation $d_{\mathrm{EM}}$ at which the electromagnetic interaction starts to be important is when the magnitude of its associated energy $\left(\lambda^{2} \mathcal{M}^{2} / d_{\mathrm{EM}}\right)$ is comparable to the kinetic energy $(2(\gamma-1) \mathcal{M}):^{2}$

$$
d_{\mathrm{EM}}=\frac{\lambda^{2} \mathcal{M}}{2(\gamma-1)}=\frac{\lambda^{2}}{4} \frac{\mathcal{M}_{\mathrm{ADM}}}{\gamma(\gamma-1)},
$$

\footnotetext{
2 The gravitational interaction starts to be important at larger separations. However, this increases the kinetic energy and only makes the conclusions stronger.
} 
where we used that $\mathcal{M}_{\mathrm{ADM}}=2 \gamma \mathcal{M}$. For separations that are much larger than this value, the bodies can be considered non-interacting, so charge does not matter. In classical physics, particles will always reach $d_{\mathrm{EM}}$, where they start to be repelled by the electrostatic force. This is not what happens for black holes, where there is another length-scale that we need to consider and that drastically alters this picture. Assuming that all the initial energy ends up in the remnant, and calling $\mathcal{R}=2 \mathcal{M}_{\mathrm{ADM}}$ its Schwarzschild radius, we expect $\mathcal{R}$ to be where generalrelativistic effects to be dominant (consider, for example, the hoop conjecture [64]). When the two initial horizons get closer than $\mathcal{R}$, they stick together as a newly formed remnant, overcoming the electrostatic repulsion. So, if $d_{\text {EM }} \ll \mathcal{R}$, electromagnetism starts to be dominant only after the formation of a common apparent horizon and charge would be unimportant. We conclude that charge does not matter when $d_{\mathrm{EM}} / \mathcal{R} \ll 1$, and, according to our simple model, $d_{\mathrm{EM}} / \mathcal{R}=\lambda^{2} /[8 \gamma(\gamma-1)]$. This value is smaller or much smaller than 1 for all $\lambda$ and $P$ we considered, consistently with the results of our numericalrelativity simulations.

Established that charge plays a subdominant role in the dynamics of the event under consideration, we can now turn to the problem of cosmic censorship. We argue that the conjecture is not violated in ultra-relativistic head-on collisions of charged black holes on the grounds that the final black hole always has $\lambda^{\text {remnant }}<1$ for any given initial charge and momentum. We tackle this problem with conservation arguments. Consider two black holes with Christodoulou mass $\mathcal{M}$, charge $\mathcal{Q}=\lambda \mathcal{M}$ boosted with Lorentz factor $\gamma$ and initial separation such that they can be considered non-interacting. Conservation of energy implies that the mass of the remnant has to be $\mathcal{M}^{\text {remnant }}=\mathcal{M}_{\mathrm{ADM}}-E_{\mathrm{GW}}-E_{\mathrm{EM}}$, where $E_{\mathrm{GW}}$ and $E_{\mathrm{EM}}$ are the energies carried away by gravitational and electromagnetic waves respectively. Let us define $\Upsilon(\gamma)=E_{\mathrm{EM}} / E_{\mathrm{GW}}$ and $Z(\gamma)=E_{\mathrm{GW}} / \mathcal{M}_{\mathrm{ADM}}$. As shown in Fig. 2, the ZFL approach provides a good approximation to $Z(\gamma)$, so we can use the expression in Eq. (3) in [3], noting that $Z(\gamma) \lesssim 0.14$ for any value of $\gamma[3,6]$. Conversely, we do not have a good formula for $\Upsilon(\gamma)$. In [40] it was found that $\Upsilon(1) \approx \lambda^{2} / 4$, and our simulations show that $\Upsilon(\gamma) \ll \lambda^{2} / 4$ even for low values of $\gamma$, in accordance with the conjecture that charge does not matter. So, assuming that the conjecture is true, $\Upsilon(\gamma)$ has to be at least bound. Dividing the equation of energy conservation by $\mathcal{M}_{\mathrm{ADM}}$ and using $\mathcal{M}^{\text {remnant }}=2 \mathcal{Q}$ / $\lambda^{\text {remnant }}$ (charge is conserved) and $\mathcal{M}_{\mathrm{ADM}}=2 \gamma \mathcal{Q} / \lambda$, we find that

$$
\lambda^{\text {remnant }}(\gamma)=\left[\frac{1}{1-(1+\Upsilon(\gamma)) Z(\gamma)}\right] \frac{\lambda}{\gamma} .
$$

Given that $\Upsilon(\gamma)$ and $Z(\gamma)$ are bound, there exists a constant $C$ such that the term in the brackets is smaller than $C$ for all $\gamma$. Hence, $\lambda^{\text {remnant }} \leq C \lambda / \gamma$, indicating that $\lambda^{\text {remnant }}$ decreases with $\gamma$. In Fig. 3, we show Eq. (3) by reporting the values of $\lambda^{\text {remnant }}$ predicted for various $\lambda$ assuming $\Upsilon(\gamma) \ll 1$. We overlay the result of our simulations with markers, which are in excellent agreement. Since in the limit of $\gamma \rightarrow \infty$, Eq. (3) predicts that $\lambda^{\text {remnant }}$ goes to zero, we find agreement with the conjecture that matter does not matter and we conclude ultra-relativistic head-on collisions of charged black holes should not be expected to form naked singularities. This result is robust and only depends on the assumption that electromagnetic waves cannot radiate away all the additional kinetic energy, as our general relativistic calculations show.

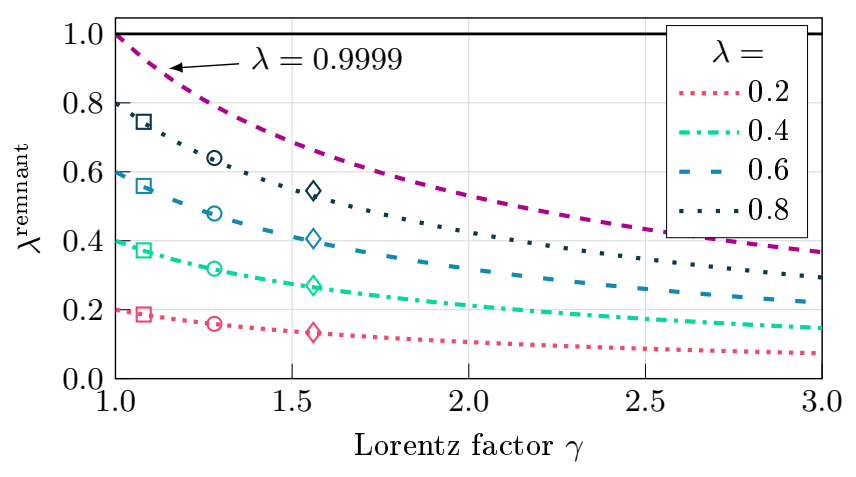

FIG. 3. Charge-to-mass ratio $\lambda^{\text {remnant }}$ for the remnant left by a merger of two equal-mass black holes with initial Lorentz factor $\gamma$ and charge-to-mass ratio $\lambda$. The curves are obtained with Eq. (3) assuming $\Upsilon(\gamma)=0$ (expected from the fact that charge does not matter in the energy emitted in these mergers) and the markers are the values from our simulations. The figure seems to hint that the only case where we can obtain an overcharged remnant is with $\lambda, \gamma \rightarrow 1$, where our approximations break down and previous studies found no violation [40].

Conclusions Ultra-relativistic collisions of black holes are fertile ground for theoretical studies in general relativity and high energy physics. In this Letter, we presented the first results on high-energy head-on mergers of charged black holes. We found that the intuition built with simple semi-classical arguments carries over to full general relativity. First, we found that charge does not play an important role, supporting the conjecture that matter does not matter. This is an important step in claiming that the conclusion holds for generic fourdimensional general-relativistic black holes. This result is also important in the context of the production of microscopic black holes in particle accelerators and cosmic rays. We also argued that, as a result, we should not expect the formation of naked singularities in this kind of event.

Given that the expectation that charge is unimportant is met even with relatively low boosts, we anticipate that varying the other variables that were not considered here (mass, impact parameter, charge, spin) will yield the same results as the uncharged case. This should be 
tested, along with expanding the current study to more extreme $\lambda$ and $P$ and increasing the accuracy. This might require enhancement in the initial data (e.g., by using better guesses for the electromagnetic fields and by lifting the assumption of conformal flatness) and a reduction in the error budget (e.g., by reducing initial data ambiguity, increasing the accuracy in the wave zone-possibly with multi-patch grids [65]-and performing interpolation of waves to infinity).

G. B. is indebted to Vasilis Paschalidis for several insightful conversations and comments on the manuscript. This research was made possible by the developers and maintainers of the open-source codes that we used. kuibit [38] uses NumPy [66], SciPy [67], and h5py [68]. This work was supported by NSF Grant PHY-1912619 to the University of Arizona, a Frontera Fellowship by the Texas Advanced Computing Center (TACC), and NASA Grant 80NSSC20K1542. Frontera [69] is founded by NSF grant OAC-1818253. Computational resources were provided by the Extreme Science and Engineering Discovery Environment (XSEDE) under grant number TG-PHY190020. XSEDE is supported by the NSF grant No. ACI-1548562. Simulations were performed on Stampede2, which is funded by the NSF through award ACI-1540931.

\section{SUPPLEMENTAL MATERIAL: COMPARISON WITH PREVIOUS STUDIES}

Our work extends previous studies [40] to the case with non-zero initial boost. In [40], it was found that charge plays an important role in the merger, while we find that at $\gamma \approx 1.1$ the effects are negligible. Our set of simulations does not capture the transition between the two regimes. To do so one would need to perform simulations with smaller $\gamma$, calculations that are computationally expensive with a setup like ours. Our simulations are at high resolution (up to $M / 550$ on the finest level) and with an initial separation of $150 \mathrm{M}$, leading to a long time to merger. To further connect our results with the ones in [40], we reproduced selected simulations in [40] (where the initial separation and resolution are smaller than the ones used in this work). Fig. 4 shows the coordinate separation for mergers of charged black holes with no initial boost. While this is not a gauge-invariant quantity, it is suggestive of what happens when $\gamma=0$ : systems with different values of charge behave differently. More specifically, Fig. 5 shows that this behavior is fully captured by the classical arguments that predict a scaling as $\sqrt{1-\lambda^{2}}$. This is the main result obtained in [40]. These two plots are in contrast with Fig. 1, where no scaling was needed to obtain overlap between the different signals.

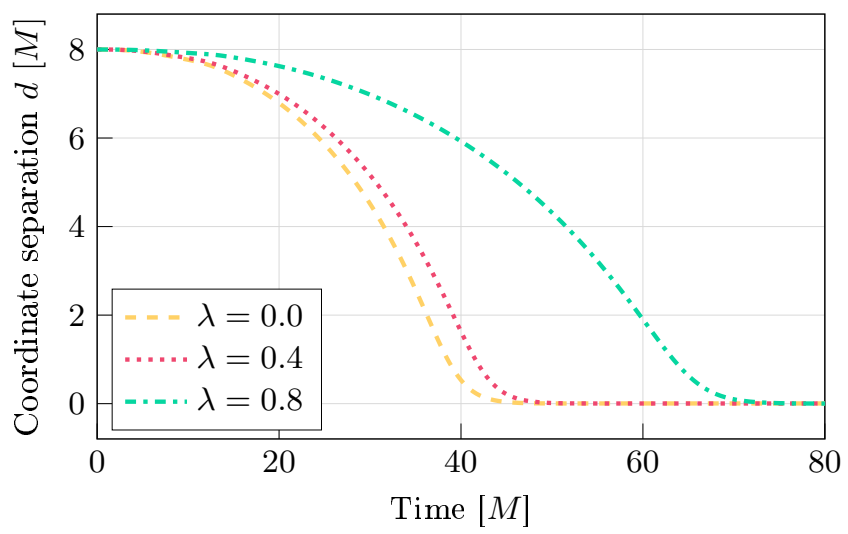

FIG. 4. Coordinate separation as a function of time for mergers of equal-mass, equal-charge black holes with initial separation of $8 M$ zero boost. The plot shows that the dynamics of the event depend on the charge-to-mass ratio $\lambda$, in contrast to what found for the case with larger initial boost (see, Fig. 1).

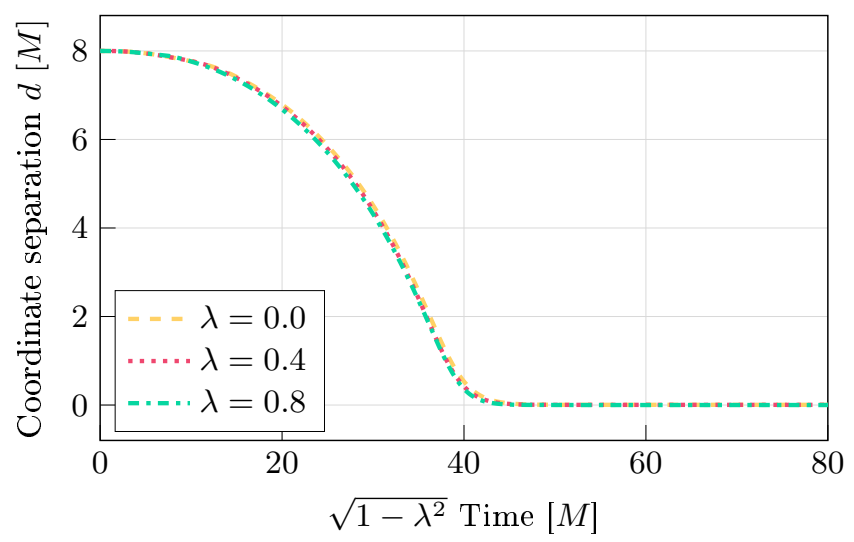

FIG. 5. Same as Fig. 4, but with time rescaled by $\sqrt{1-\lambda^{2}}$. This is the same result obtained in [40], where it was found that charge matters for mergers with zero initial velocity.

\section{SUPPLEMENTAL MATERIAL: ERROR ESTIMATE}

In our study, we have both numerical and systematic sources of error. Here, we discuss them and provide an estimate of the overall accuracy of our simulations. Note that we do not need an accurate estimate of the error, since the results we presented are not affected by this.

The results of this Letter hinge upon comparing simulations with same value of $P$ and different value of $\lambda$. This comparison is not straightforward for several reasons. First, the assumptions that go in the initial data (mainly the conformal flatness and the initial electromagnetic fields) lead to initial black holes that are not perfectly in equilibrium. Hence, the initial data relaxes to a new configuration with different values of mass and mo- 
mentum. ${ }^{3}$ We report how the irreducible mass evolves for a five representative cases in Fig. 6 For the simulations explored in this Letter, the change is always below $1.5 \%$, providing an estimate of the accuracy of the initial mass and momentum. For a fixed momentum $P$, simulations behave differently depending on $\lambda$, with higher charges starting further from equilibrium. The assumptions in the initial data also limit the maximum boost attainable, as for larger values of $P$, the initial data relaxes to a new configuration with a smaller momentum. Second, in our simulations, we fix the coordinate distance between the two punctures, which, by definition, is a gauge-dependent quantity. Simulations have different coordinates, so it is not possible to compare them directly. In [40], a more robust way to compare different simulations of charged black holes is used and the proper distance between the horizons is computed. It was found (Table I in [40]) that the difference for different values of charge is at the level of the percent. While the initial data are different, we expect similar results to hold here. Moreover, since the black holes start from a distance of $150 \mathrm{M}$, they are almost isolated (with error at the level of the percent). Third, initial data contains junk radiation, especially electromagnetic (due to the choice of initial electromagnetic fields, which is not well adapted to cases with high boost). For large charge and boosts, this radiation can be up to a few percent of the total energy in the spacetime and is another source of error and limit to our study. Because of the different amount of junk radiation and the interaction energy, simulations will have slightly different ADM mass for a fixed value of $P$, with a maximum variation of $2 \%$. This directly affects the denominator in Fig. 2. These three effects introduce a fundamental systematic error of order of percent in our work that would still be present in the limit of infinite numerical resolution.

The second source of error is due to the finite resolution of our simulations and the finite extraction radius. We estimate both by considering different extraction radii and performing selected simulations at higher resolution. In Fig. 7, we compare the extracted $\Psi_{4}^{20}$ for three selected cases. The figure shows that there is convergence, but the amount and properties of noise depend on the resolution and the extraction radius. This is a well-known and common feature in numerical-relativity calculations [71]. Electromagnetic waves have more noise, but they are always subdominant, so they do not affect the overall error. We obtain an estimate of the accuracy of our calculation by comparing the amount of total energy radiated for different resolutions and extraction radii. Combining all

\footnotetext{
3 One could start the simulation, let the initial data relax, and measure the new mass and momentum. However, due to the lack of a well-defined notion of quasi-local linear momentum [60, 70], this task is not trivial and beyond the goals of this Letter.
}

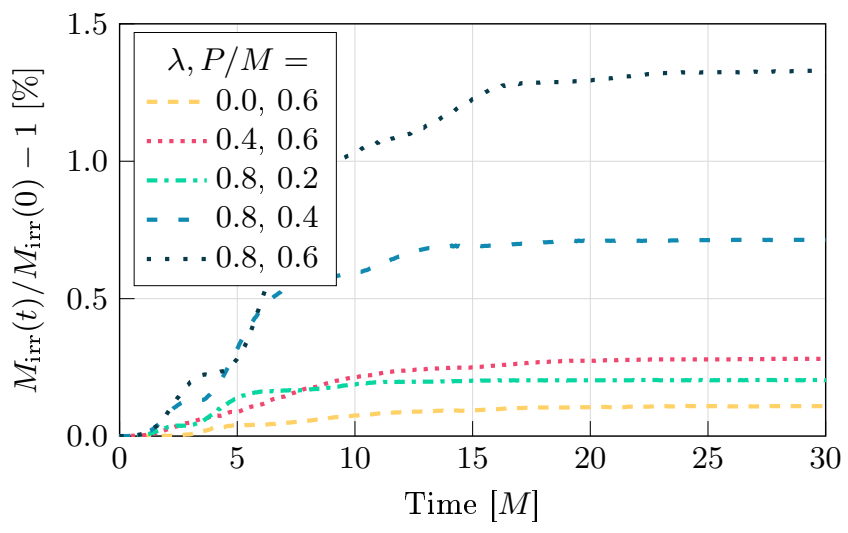

FIG. 6. Variation in the irreducible mass of one of the horizons as measured by QuasiLocalMeasuresEM in the first $30 \mathrm{M}$ of evolution. We use this quantity to asses the quality of the initial data and the relaxation time. Our assumption on conformal flatness and the choice of initial electromagnetic fields limits the maximum linear momentum that can be generated. Higher charge-to-mass ratio $\lambda$ and momentum $P$ lead to initial data further from equilibrium. The masses does not grow past the time plotted here, so we can see that the relaxation time is of order of $10 \mathrm{M}$.

sources of error, we estimate that the error in the energy lost by waves to be of order $10 \%$. On the other hand, the horizon properties are stable and show excellent degree of convergence (the final properties differ by less than $0.01 \%$ ). So, we assume that the dominant source of error for the remnant properties is the intrinsic uncertainty in the initial data. Therefore, we estimate the error in the identification of the parameters of the final remnant to be a few percent.

* gabrielebozzola@email.arizona.edu

[1] U. Sperhake, Numerical relativity: the role of black holes in gravitational wave physics, astrophysics and highenergy physics, General Relativity and Gravitation 46, 1689 (2014).

[2] M. W. Choptuik, L. Lehner, and F. Pretorius, Probing Strong Field Gravity Through Numerical Simulations, arXiv e-prints , arXiv:1502.06853 (2015), arXiv:1502.06853 [gr-qc].

[3] U. Sperhake, V. Cardoso, F. Pretorius, E. Berti, and J. A. González, High-Energy Collision of Two Black Holes, Physical Review Letters 101, 161101 (2008), arXiv:0806.1738 [gr-qc].

[4] M. Shibata, H. Okawa, and T. Yamamoto, High-velocity collision of two black holes, Phys. Rev. D 78, 101501 (2008), arXiv:0810.4735 [gr-qc].

[5] U. Sperhake, V. Cardoso, F. Pretorius, E. Berti, T. Hinderer, and N. Yunes, Cross Section, Final Spin, and Zoom-Whirl Behavior in High-Energy Black-Hole Collisions, Physical Review Letters 103, 131102 (2009), arXiv:0907.1252 [gr-qc]. 


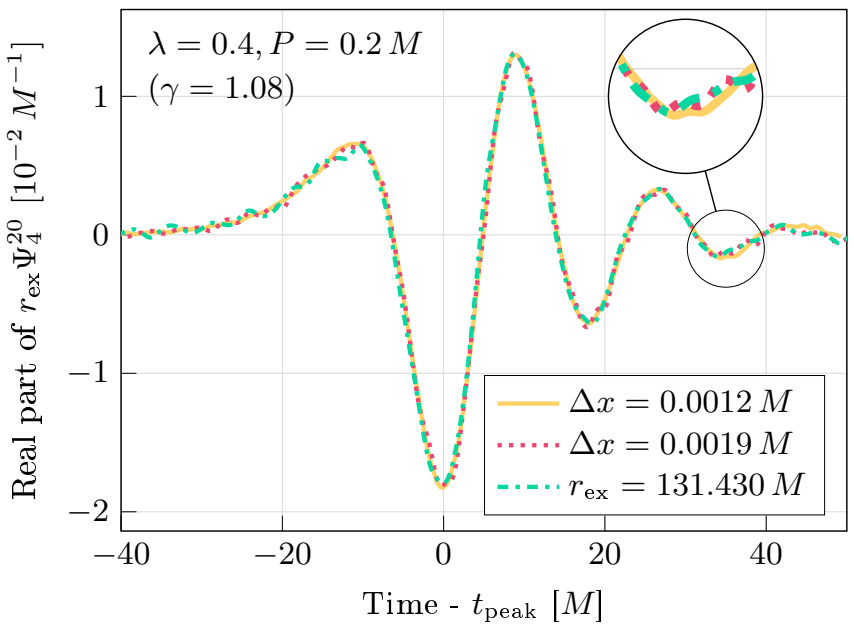

FIG. 7. Comparison between the $l=2, m=0$ mode of the Newman-Penrose scalar $\Psi_{4}$ for simulations with different resolutions and extracted at different radii $r_{\mathrm{ex}}\left(r_{\mathrm{ex}}=97.143 \mathrm{M}\right)$. The plot shows good convergence, up to a well-known highfrequency noise [71] (as shown in the inset). We obtain an error estimate comparing the total energy radiated away for simulations with different extraction radii and/or resolution.

[6] J. Healy, I. Ruchlin, C. O. Lousto, and Y. Zlochower, High energy collisions of black holes numerically revisited, Phys. Rev. D 94, 104020 (2016), arXiv:1506.06153 [gr-qc].

[7] U. Sperhake, V. Cardoso, C. D. Ott, E. Schnetter, and H. Witek, Collisions of unequal mass black holes and the point particle limit, Phys. Rev. D 84, 084038 (2011), arXiv:1105.5391 [gr-qc].

[8] U. Sperhake, E. Berti, V. Cardoso, F. Pretorius, and N. Yunes, Superkicks in ultrarelativistic encounters of spinning black holes, Phys. Rev. D 83, 024037 (2011), arXiv:1011.3281 [gr-qc].

[9] R. Gold and B. Brügmann, Eccentric black hole mergers and zoom-whirl behavior from elliptic inspirals to hyperbolic encounters, Phys. Rev. D 88, 064051 (2013), arXiv:1209.4085 [gr-qc].

[10] U. Sperhake, E. Berti, V. Cardoso, and F. Pretorius, Gravity-dominated unequal-mass black hole collisions, Phys. Rev. D 93, 044012 (2016), arXiv:1511.08209 [grqc].

[11] U. Sperhake, W. Cook, and D. Wang, High-energy collision of black holes in higher dimensions, Phys. Rev. D 100, 104046 (2019), arXiv:1909.02997 [gr-qc].

[12] T. Andrade, R. Emparan, D. Licht, and R. Luna, Black hole collisions, instabilities, and cosmic censorship violation at large D, Journal of High Energy Physics 2019, 99 (2019), arXiv:1908.03424 [hep-th].

[13] T. Andrade, P. Figueras, and U. Sperhake, Violations of Weak Cosmic Censorship in Black Hole collisions, arXiv e-prints , arXiv:2011.03049 (2020), arXiv:2011.03049 [hep-th].

[14] G. 't Hooft, Graviton dominance in ultra-high-energy scattering, Physics Letters B 198, 61 (1987).

[15] D. Amati, M. Ciafaloni, and G. Veneziano, Superstring collisions at planckian energies, Physics Letters B 197, 81 (1987).
[16] T. Banks and W. Fischler, A Model for High Energy Scattering in Quantum Gravity, arXiv e-prints , hepth/9906038 (1999), arXiv:hep-th/9906038 [hep-th].

[17] U. Sperhake, E. Berti, V. Cardoso, and F. Pretorius, Universality, Maximum Radiation, and Absorption in HighEnergy Collisions of Black Holes with Spin, Physical Review Letters 111, 041101 (2013), arXiv:1211.6114 [gr-qc].

[18] M. W. Choptuik and F. Pretorius, Ultrarelativistic Particle Collisions, Phys. Rev. Lett. 104, 111101 (2010), arXiv:0908.1780 [gr-qc].

[19] W. E. East and F. Pretorius, Ultrarelativistic Black Hole Formation, Phys. Rev. Lett. 110, 101101 (2013), arXiv:1210.0443 [gr-qc].

[20] L. Rezzolla and K. Takami, Black-hole production from ultrarelativistic collisions, Classical and Quantum Gravity 30, 012001 (2013), arXiv:1209.6138 [gr-qc].

[21] F. Pretorius and W. E. East, Black hole formation from the collision of plane-fronted gravitational waves, Phys. Rev. D 98, 084053 (2018), arXiv:1807.11562 [gr-qc].

[22] H. Witek, V. Cardoso, C. Herdeiro, A. Nerozzi, U. Sperhake, and M. Zilhão, Black holes in a box: Toward the numerical evolution of black holes in AdS space-times, Phys. Rev. D 82, 104037 (2010), arXiv:1004.4633 [hepth].

[23] H. Okawa, K.-I. Nakao, and M. Shibata, Is superPlanckian physics visible? Scattering of black holes in 5 dimensions, Phys. Rev. D 83, 121501 (2011), arXiv:1105.3331 [gr-qc].

[24] M. Cavaglià, R. Godang, L. Cremaldi, and D. Summers, Catfish: A Monte Carlo simulator for black holes at the LHC, Computer Physics Communications 177, 506 (2007), arXiv:hep-ph/0609001 [hep-ph].

[25] D.-C. Dai, G. Starkman, D. Stojkovic, C. Issever, E. Rizvi, and J. Tseng, BlackMax: A black-hole event generator with rotation, recoil, split branes, and brane tension, Phys. Rev. D 77, 076007 (2008), arXiv:0711.3012 [hep-ph].

[26] J. A. Frost, J. R. Gaunt, M. O. P. Sampaio, M. Casals, S. R. Dolan, M. A. Parker, and B. R. Webber, Phenomenology of production and decay of spinning extradimensional black holes at hadron colliders, Journal of High Energy Physics 2009, 014 (2009), arXiv:0904.0979 [hep-ph].

[27] R. M. Wald, General relativity (Chicago Univ. Press, Chicago, IL, 1984).

[28] I. Ruchlin, J. Healy, C. O. Lousto, and Y. Zlochower, Puncture Initial Data for Black-Hole Binaries with High Spins and High Boosts, Phys. Rev. D95, 024033 (2017), arXiv:1410.8607 [gr-qc].

[29] D. Christodoulou and R. Ruffini, Reversible transformations of a charged black hole, Phys. Rev. D 4, 3552 (1971).

[30] G. Bozzola and V. Paschalidis, Initial data for general relativistic simulations of multiple electrically charged black holes with linear and angular momenta, Phys. Rev. D 99, 104044 (2019), arXiv:1903.01036 [gr-qc].

[31] R. L. Arnowitt, S. Deser, and C. W. Misner, The dynamics of general relativity, General Relativity and Gravitation 40, 1997 (2008), arXiv:gr-qc/0405109.

[32] R. Arnowitt, S. Deser, and C. W. Misner, Republication of: The dynamics of general relativity, General Relativity and Gravitation 40, 1997 (2008).

[33] M. Alcubierre, Introduction to 3+1 Numerical Relativity (Oxford University Press, UK, 2008). 
[34] T. W. Baumgarte and S. L. Shapiro, Numerical Relativity: Solving Einstein's Equations on the Computer (Cambridge University Press, 2010).

[35] M. Shibata, Numerical Relativity (World Scientific Publishing Co, 2016).

[36] F. Löffler, J. Faber, E. Bentivegna, T. Bode, P. Diener, R. Haas, I. Hinder, B. C. Mundim, C. D. Ott, E. Schnetter, G. Allen, M. Campanelli, and P. Laguna, The Einstein Toolkit: A Community Computational Infrastructure for Relativistic Astrophysics, Class. Quantum Grav. 29, 115001 (2012), arXiv:1111.3344 [gr-qc].

[37] Z. Etienne, S. R. Brandt, P. Diener, W. E. Gabella, M. Gracia-Linares, R. Haas, A. Kedia, M. Alcubierre, D. Alic, G. Allen, M. Ansorg, M. Babiuc-Hamilton, L. Baiotti, W. Benger, E. Bentivegna, S. Bernuzzi, T. Bode, G. Bozzola, B. Brendal, B. Bruegmann, M. Campanelli, F. Cipolletta, G. Corvino, S. Cupp, R. D. Pietri, H. Dimmelmeier, R. Dooley, N. Dorband, M. Elley, Y. E. Khamra, J. Faber, T. Font, J. Frieben, B. Giacomazzo, T. Goodale, C. Gundlach, I. Hawke, S. Hawley, I. Hinder, E. A. Huerta, S. Husa, S. Iyer, D. Johnson, T. Kellermann, A. Knapp, M. Koppitz, P. Laguna, G. Lanferman, F. Löffler, J. Masso, L. Menger, A. Merzky, J. M. Miller, M. Miller, P. Moesta, P. Montero, B. Mundim, A. Nerozzi, S. C. Noble, C. Ott, R. Paruchuri, D. Pollney, D. Radice, T. Radke, C. Reisswig, L. Rezzolla, D. Rideout, M. Ripeanu, L. Sala, J. A. Schewtschenko, E. Schnetter, B. Schutz, E. Seidel, E. Seidel, J. Shalf, K. Sible, U. Sperhake, N. Stergioulas, W.-M. Suen, B. Szilagyi, R. Takahashi, M. Thomas, J. Thornburg, M. Tobias, A. Tonita, P. Walker, M.-B. Wan, B. Wardell, L. Werneck, H. Witek, M. Zilhão, B. Zink, and Y. Zlochower, The einstein toolkit (2021), to find out more, visit http://einsteintoolkit.org.

[38] G. Bozzola, kuibit: Analyzing Einstein Toolkit simulations with Python, The Journal of Open Source Software 6, 3099 (2021), arXiv:2104.06376.

[39] G. Bozzola and V. Paschalidis, Numerical-relativity simulations of the quasicircular inspiral and merger of nonspinning, charged black holes: Methods and comparison with approximate approaches, Phys. Rev. D 104, 044004 (2021), arXiv:2104.06978 [gr-qc].

[40] M. Zilhão, V. Cardoso, C. Herdeiro, L. Lehner, and U. Sperhake, Collisions of charged black holes, Phys. Rev. D 85, 124062 (2012), arXiv:1205.1063 [gr-qc].

[41] M. Zilhão, V. Cardoso, C. Herdeiro, L. Lehner, and U. Sperhake, Collisions of oppositely charged black holes, Phys. Rev. D 89, 044008 (2014), arXiv:1311.6483 [gr-qc].

[42] J. W. York, Gravitational Degrees of Freedom and the Initial-Value Problem, Physical Review Letters 26, 1656 (1971).

[43] J. M. Bowen, Inversion symmetric initial data for $\mathrm{N}$ charged black holes., Annals of Physics 165, 17 (1985).

[44] M. Alcubierre, J. C. Degollado, and M. Salgado, EinsteinMaxwell system in 3+1 form and initial data for multiple charged black holes, Phys. Rev. D 80, 104022 (2009), arXiv:0907.1151 [gr-qc].

[45] M. Ansorg, B. Brügmann, and W. Tichy, Single-domain spectral method for black hole puncture data, Phys. Rev. D 70, 064011 (2004), gr-qc/0404056.

[46] H. Witek and M. Zilhão, Canuda code (2015).

[47] H. Witek, M. Zilhao, G. Bozzola, M. Elley, G. Ficarra, T. Ikeda, N. Sanchis-Gual, and H. Silva, Canuda: a public numerical relativity library to probe fundamental physics (2021).

[48] U. Sperhake, Binary black-hole evolutions of excision and puncture data, Phys. Rev. D 76, 104015 (2007), grqc/0606079.

[49] M. Zilhão, H. Witek, and V. Cardoso, Nonlinear interactions between black holes and Proca fields, Classical Quant. Grav. 32, 234003 (2015), arXiv:1505.00797 [grqc].

[50] M. Shibata and T. Nakamura, Evolution of threedimensional gravitational waves: Harmonic slicing case, Phys. Rev. D 52, 5428 (1995).

[51] T. W. Baumgarte and S. L. Shapiro, Numerical integration of einstein's field equations, Phys. Rev. D 59, 024007 (1998).

[52] M. Alcubierre, B. Brügmann, P. Diener, M. Koppitz, D. Pollney, E. Seidel, and R. Takahashi, Gauge conditions for long term numerical black hole evolutions without excision, Phys. Rev. D 67, 084023 (2003), arXiv:grqc/0206072.

[53] J. R. van Meter, J. G. Baker, M. Koppitz, and D.-I. Choi, How to move a black hole without excision: Gauge conditions for the numerical evolution of a moving puncture, Phys. Rev. D 73, 124011 (2006), gr-qc/0605030.

[54] I. Hinder et al., Error-analysis and comparison to analytical models of numerical waveforms produced by the NRAR Collaboration, Class. Quant. Grav. 31, 025012 (2014), arXiv:1307.5307 [gr-qc].

[55] E. Schnetter, S. H. Hawley, and I. Hawke, Evolutions in 3D numerical relativity using fixed mesh refinement, Class. Quantum Grav. 21, 1465 (2004), arXiv:gr-qc/0310042.

[56] J. Thornburg, A Fast Apparent-Horizon Finder for 3-Dimensional Cartesian Grids in Numerical Relativity, Class. Quantum Grav. 21, 743 (2004), arXiv:grqc/0306056.

[57] O. Dreyer, B. Krishnan, D. Shoemaker, and E. Schnetter, Introduction to isolated horizons in numerical relativity, Phys. Rev. D 67, 024018 (2003), gr-qc/0206008.

[58] A. Ashtekar, C. Beetle, O. Dreyer, S. Fairhurst, B. Krishnan, J. Lewandowski, and J. Wiśniewski, Generic Isolated Horizons and Their Applications, Phys. Rev. Lett. 85, 3564 (2000), gr-qc/0006006.

[59] A. Ashtekar, C. Beetle, and J. Lewandowski, Mechanics of rotating isolated horizons, Phys. Rev. D 64, 044016 (2001), gr-qc/0103026.

[60] A. Ashtekar and B. Krishnan, Isolated and Dynamical Horizons and Their Applications, Living Reviews in Relativity 7, 10 (2004), gr-qc/0407042.

[61] E. Newman and R. Penrose, An Approach to Gravitational Radiation by a Method of Spin Coefficients, Journal of Mathematical Physics 3, 566 (1962).

[62] G. Bozzola and V. Paschalidis, General Relativistic Simulations of the Quasicircular Inspiral and Merger of Charged Black Holes: GW150914 and Fundamental Physics Implications, Phys. Rev. Lett. 126, 041103 (2021), arXiv:2006.15764 [gr-qc].

[63] L. Smarr, Gravitational radiation from distant encounters and from head-on collisions of black holes: The zerofrequency limit, Phys. Rev. D 15, 2069 (1977).

[64] K. S. Thorne, Nonspherical Gravitational Collapse-A Short Review, in Magic Without Magic: John Archibald Wheeler, edited by J. R. Klauder (1972) p. 231.

[65] D. Pollney, C. Reisswig, E. Schnetter, N. Dorband, and P. Diener, High accuracy binary black hole simulations with an extended wave zone, Phys. Rev. D 83, 044045 
(2011), arXiv:0910.3803 [gr-qc].

[66] C. R. Harris, K. J. Millman, S. J. van der Walt, R. Gommers, P. Virtanen, D. Cournapeau, E. Wieser, J. Taylor, S. Berg, N. J. Smith, R. Kern, M. Picus, S. Hoyer, M. H. van Kerkwijk, M. Brett, A. Haldane, J. F. del Río, M. Wiebe, P. Peterson, P. Gérard-Marchant, K. Sheppard, T. Reddy, W. Weckesser, H. Abbasi, C. Gohlke, and T. E. Oliphant, Array programming with NumPy, Nature 585, 357 (2020).

[67] P. Virtanen, R. Gommers, T. E. Oliphant, M. Haberland, T. Reddy, D. Cournapeau, E. Burovski, P. Peterson, W. Weckesser, J. Bright, S. J. van der Walt, M. Brett, J. Wilson, K. Jarrod Millman, N. Mayorov, A. R. J. Nelson, E. Jones, R. Kern, E. Larson, C. Carey, I. Polat, Y. Feng, E. W. Moore, J. Vand erPlas, D. Laxalde, J. Perktold, R. Cimrman, I. Henriksen, E. A. Quin- tero, C. R. Harris, A. M. Archibald, A. H. Ribeiro, F. Pedregosa, P. van Mulbregt, and S. . . Contributors, SciPy 1.0: Fundamental Algorithms for Scientific Computing in Python, Nature Methods 17, 261 (2020).

[68] A. Collette, Python and HDF5 (O'Reilly, 2013).

[69] D. Stanzione, J. West, R. T. Evans, T. Minyard, O. Ghattas, and D. K. Panda, Frontera: The evolution of leadership computing at the national science foundation, in Practice and Experience in Advanced Research Computing, PEARC '20 (Association for Computing Machinery, New York, NY, USA, 2020) p. 106-111.

[70] B. Krishnan, Isolated Horizons in Numerical Relativity, Ph.D. thesis, The Pennsylvania State University (2002).

[71] Y. Zlochower, M. Ponce, and C. O. Lousto, Accuracy issues for numerical waveforms, Phys. Rev. D 86, 104056 (2012), arXiv:1208.5494 [gr-qc]. 